\title{
The Action Potential simulated by Mass Action Law Modelación de Potenciales de Acción generados mediante la Ley de Acción de Masas
}

\section{The Action Potential simulated by Mass Action Law Modeling of Action Potentials generated by Mass Action Law}

DOI: $10.46932 / \mathrm{sfjdv2n5-136}$

Received in: Oct 1st, 2021

Accepted in: Dec 30th, 2021

\author{
Alondra Albarado Ibañez \\ Centro de investigación de Fisicoquímica de los materiales Instituto de Ciencias, Benemérita \\ Universidad Autónoma de Puebla. \\ Apartado postal 1152, 72000, Puebla Pue. México. \\ E-mail: alondra.albarado@correo.buap.mx
}

Areli Montes Pérez

Facultad de Ciencias Físico Matemáticas Benemérita Universidad Autónoma de Puebla. Apartado postal 1152, 72000 Puebla Pue. México.

E-mail: arelimp@fcfm.buap.mx

Julia Aguirre Sánchez

Facultad de Ciencias Físico Matemáticas Benemerita Univesidad Autónoma de Puebla. Apartado postal 1152, 72000 Puebla Puebla Mexico.

E-mail: julia.aguirre1911@gmail.com

Javier González Medrano

Facultad de Ciencias Físico Matemáticas Benemerita Univesidad Autónoma de Puebla. Apartado postal 1152, 72000 Puebla Pue. Mexico

\section{Jorge Torres Jácome}

Departamento de Matemáticas del Instituto de Ciencias, Benemérita Universidad Autónoma de Puebla. Apartado postal 1152, 72000, Puebla Pue. Mexico.

E-mail: jorge.torres@ correo.buap.mx

\section{Julián Torres Jácome}

Grupo de Fisiopatologia Cardiovascular, Benemérita Universidad Autónoma de Puebla. Apartado postal

1152, 72000, Puebla Pue. Mexico.

E-mail: jtorresjacome@gmail.com

\section{RESUMEN}

En este trabajo se presenta un modelo matemático para potenciales de acción (PA), similar al modelo de Hodgkin y Huxley, utilizando la Ley de Acción de Masas para determinar las corrientes iónicas que atraviesan la membrana en cada célula excitable. Este modelo permite visualizar que la cinética de los canales iónicos (las velocidades de transición de un estado a otro), determina el curso temporal de las corrientes. Además, resalta que los tipos y la intensidad de las corrientes activadas en la membrana celular, determinan la morfología de los PA. Por lo anterior, a cada potencial de acción se le asociaron corrientes iónicas especificas, con esta condición, el modelo generó un sistema de ecuaciones diferenciales cuya 
solución es la función $\mathrm{V}=\mathrm{V}(\mathrm{t})$, los resultados mostraron que esta función simula a los PA registrados experimentalmente.

Palabras clave: Ley de acción de masas, Potencial de acción, Hodgkin y Huxley.

\begin{abstract}
In this work, we simulated action potentials with a mathematical model similarly to the Hodgkin and Huxley model, only we are using the Mass Action Law. This model showed that the transition of velocities from one state to another of the channels, they are establishing the time course of the currents. Also, the morphology of the action potentials depend on the types and intensity of the currents activated in the cell membrane. Therefore, specific ionic currents were associated to each action potential, with this condition, the model generated a system of differential equations whose solution is the function $V=V(t)$, the results showed that this function simulates the action potentials in experimental record.
\end{abstract}

Keywords: Law of mass action, Action potential, Hodgkin and Huxley.

\title{
1 INTRODUCCIÓN
}

Los PA son cambios transitorios de voltaje que se propagan en las células excitables ${ }^{1}$, tales como neuronas, células del corazón, células del musculo esquelético, algunas células endócrinas. Hay diferentes morfologías de PA, debido a el tipo y la cantidad de corriente que atraviesa la membrana celular. El potencial de acción es importante en procesos fisiológicos como la transmisión de la información entre células neuronales, acoplar la contracción muscular, la secreción de células endocrinas ${ }^{2}$. Entender la generación del potencial de acción y cambios en su morfología es importante, ya que nos permiten explicar el origen de las patologías. La generación de modelos matemáticos que enfaticen sobre las causas de los cambios en las morfologías de los PA son de gran utilidad ya que podrían explicar la causa de diferentes patologías. El trabajo mas notable que modela y describe el potencial de acción es el de Hodgkin y Hulxey ( $\mathrm{H}$ y $\mathrm{H})$ publicado en $1952^{3}$.

\section{MODELO TEÓRICO}

Para explicar el modelo tenemos que considerar lo siguiente: las células están delimitadas por una membrana que consiste en una bicapa de fosfolípidos lo que la hace totalmente impermeable, dentro de ella existe una solución con diferentes concentraciones de sales como $\mathrm{Na}^{+}, \mathrm{K}^{+}, \mathrm{Ca}^{++}, \mathrm{Cl}^{--}$, haciendo que el interior de las células se comporte como un conductor, fuera de ella existe también una solución con diferentes concentraciones de estas sales, es decir, se puede modelar a una célula como un conductor rodeado de un aislante y después rodeado de otro conductor. Lo anterior da a la célula, la función de un capacitor eléctrico. En la membrana celular existen proteínas transmembranales o canales iónicos que a ciertos voltajes se hacen permeables a un ion especifico generando una conductancia al ion $\left(\mathrm{G}_{(\mathrm{ion})}\right)$. La $\mathrm{G}_{(\mathrm{ion})}$ es dependiente de voltaje, las células no se comportan como un circuito Óhmico. La concentración 
dentro de la célula del ion potasio es del orden de $120 \mathrm{mM}$ y fuera de ella es de $5 \mathrm{mM}^{4}$ esta diferencia de concentraciones genera una diferencia de voltaje entre dentro y fuera de la célula del orden de $-70 \mathrm{mV}$ en condiciones de reposo, es decir cuando la célula es solo permeable al ion $\mathrm{K}^{+}$.

\subsection{TEJIDOS EXCITABLES}

Las células que forman un tejido excitable tienen en su membrana canales iónicos voltaje dependientes, de tal forma que ante un estimulo adecuado se produce un aumento del voltaje, los canales se activan y producen corrientes iónicas que atraviesan la membrana aumentando su voltaje y produciendo un aumento transitorio que se propaga por toda su membrana. Es decir, las células excitables son aquellas que en respuesta a un estimulo adecuado producen un potencial de acción.

\subsection{POTENCIAL DE ACCIÓN}

La hipótesis propuesta por $\mathrm{H}$ y $\mathrm{H}$ fue la siguiente "la membrana tiene canales que permiten el paso de iones en la dirección que determina su potencial electroquímico”. Este movimiento iónico produce corrientes eléctricas que cambian el voltaje de la membrana produciendo el potencial de acción.

Partiendo del potencial de reposo, cuando se estimula una célula nerviosa, en un axón de calamar gigante, la conductancia al ion sodio $\left(\mathrm{G}_{(\mathrm{Na})}\right)$ aumenta, este aumento permite que el voltaje de la membrana incremente, lo que a su vez aumenta la $\mathrm{G}_{(\mathrm{na})}$ ya que esta conductancia depende del voltaje y del tiempo $\mathrm{G}_{(\mathrm{Na})}=\mathrm{G}_{(\mathrm{Na})}(\mathrm{t}, \mathrm{V})$. Simultáneamente la conductancia del ion potasio es función del tiempo y del voltaje $\mathrm{G}_{(\mathrm{k})}=\mathrm{G}_{(\mathrm{k})}(\mathrm{t}, \mathrm{V})$, lo que permite que el potasio salga de la célula, disminuyendo el voltaje de la membrana. Estos movimientos iónicos permiten que el voltaje regrese hasta el valor de reposo de la membrana. Las funciones $\mathrm{G}_{(\mathrm{na})}=\mathrm{G}_{(\mathrm{na})}(\mathrm{t}, \mathrm{V})$ y $\mathrm{G}_{(\mathrm{k})}=\mathrm{G}_{(\mathrm{k})}(\mathrm{t}, \mathrm{V})$ determinan el potencial de acción. Para lograr esto $\mathrm{H}$ y $\mathrm{H}$ suponen que la membrana se representa como un circuito RC, como se muestra en la figura 1.

Figura1 Circuito eléctrico de la membrana propuesto por Hodking y Huxley (modificado de HyH)

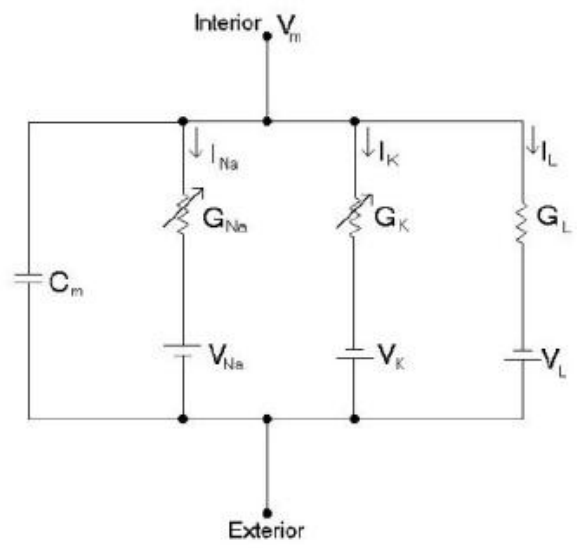


Las proteínas de membrana o canales iónicos permiten el paso selectivo de los iones, lo que representa las resistencias en el circuito ver figura 1. Con esta suposición toda corriente que atraviesa la membrana se divide en, la que pasa por el capacitor Ic y la que pasa por los canales las que se llaman corrientes iónicas. La corriente iónica a su vez se divide en el tipo de ion que pasa por el canal es decir de $\mathrm{I}_{(\mathrm{Na})}, \mathrm{I}_{(\mathrm{k})} \mathrm{y}$ en el caso de que existan mas variedad de canales se suman las corrientes. Suponiendo como lo demostraron Cole y Curtis $1939^{5}$, que la capacitancia es constante, la corriente se puede representar como en la ecuación 1.

$$
I=I_{I}+C_{m} \frac{d(V(t))}{d t} \quad \text { Ecuación } 1
$$

Donde I es la densidad de corriente total que incluye el estimulo que se le aplica a la célula un pulso cuadrado de corta duración, para iniciar el potencial de acción, $\mathrm{I}_{\mathrm{i}}$ es la corriente iónica total, $\mathrm{C}_{\mathrm{m}}$ es la capacitancia de la célula V(t) es el voltaje de membrana.

En el modelo de $\mathrm{H}$ y $\mathrm{H}$ consideran solo las corrientes de sodio $\left(\mathrm{I}_{\mathrm{na}}\right)$, potasio $\left(\mathrm{I}_{\mathrm{k}}\right)$ y una corriente que le llamaron corriente de fuga $\left(\mathrm{I}_{1}\right)$. Es decir

$$
I_{i}=I_{n a}+I_{k}+I_{l} \quad \text { ecuación } 2
$$

Cada corriente se puede determinar usando la ley de Ohm como

$$
I_{j}=G_{i}\left(V_{m}-V_{j}\right) . \quad \text { Ecuación } 3
$$

Donde $\mathrm{G} i$ es la conductancia a el ion $i$ las cuales son funciones del tiempo y del voltaje, $\mathrm{V}_{\mathrm{m}}$ es el voltaje de la membrana y $\mathrm{V}_{\mathrm{j}}$ es el voltaje de equilibrio del ion $\mathrm{j}$.

De la ecuación 1 y con las consideraciones anteriores se obtiene:

$$
C_{m} \frac{d(V(t))}{d t}=I-\left[G_{n a} m^{3} h\left(V(t)-V_{n a}\right)+G_{k} n^{4}\left(V(t)-V_{k}\right)+G_{l}\left(V(t)-V_{l}\right)\right] \text { ecuación } 4
$$

En este modelo suponían que el canal de potasio tiene dos estados uno conductor $\mathrm{n}$ y otro no conductor 1-n, que el canal de sodio tiene un estado conductor m, otro estado no conductor 1-m, y otro estado diferente no conductor que llamaron inactivado h. Consideran que los estados son independientes y que están regidos por las siguientes ecuaciones: 


$$
\begin{aligned}
& \frac{d n}{d t}=\alpha_{n}(V)(1-n)-\beta_{n}(V) n . \\
& \frac{d m}{d t}=\alpha_{m}(V)(1-m)-\beta_{m}(V) m \\
& \frac{d h}{d t}=\alpha_{h}(V)(1-h)-\beta_{h}(V) h .
\end{aligned}
$$

Donde $\alpha_{i} \quad \beta_{i}$ representan la velocidad de transición de un estado a otro de los canales. Al resolver este sistema $\mathrm{H}$ y $\mathrm{H}$ encuentran $\mathrm{V}(\mathrm{t})$ como solución y la solución da un potencial de acción solo cuando consideran $n^{4} \mathrm{y} \mathrm{m}^{3}$.

\subsection{POTENCIALES DE ACCIÓN MEDIANTE LA LEY DE ACCIÓN DE MASAS}

En este trabajo se plantea una modificación al sistema de ecuaciones 5, consideramos que el paso de un estado a otro del canal es como un sistema dinámico, es decir, que los canales están siempre pasando del estado cerrado al abierto y del abierto al inactivado, que este paso se da como una reacción química (ecuación 6) que es regida por la ley de acción de masa.

$$
C_{1} \stackrel{\alpha_{1}(V, t)}{\stackrel{\beta_{1}(V, t)}{\longrightarrow}} C_{2} \underset{\beta_{2}(V, t)}{\stackrel{\alpha_{2}(V, t)}{\longrightarrow}} C_{3} \stackrel{\alpha_{3}(V, t)}{\stackrel{\alpha_{3}(V, t)}{\longrightarrow}} O \underset{\delta(V, t)}{\stackrel{\gamma(V, t)}{\longrightarrow} I} \quad \text { ecuacion } 6
$$

Consideramos como $\mathrm{H}$ y $\mathrm{H}$ que las $\gamma \delta \alpha_{i} \quad y \beta_{i}$ dependen del tiempo y del voltaje. Con esta consideración el sistema 5 se sustituye por el sistema de ecuaciones $7^{6}$.

$$
\begin{aligned}
& \frac{d C_{1}(t)}{d t}=-\alpha_{1} C_{1}(t)+\beta_{1} C_{2}(t) \\
& \frac{d C_{2}(t)}{d t}=\alpha_{1} C_{1}(t)-\beta_{1} C_{2}(t)-\alpha_{2} C_{2}(t)+\beta_{2} C_{3}(t) \\
& \frac{d C_{3}(t)}{d t}=\alpha_{2} C_{2}(t)-\beta_{2} C_{3}(t)-\alpha_{3} C_{3}(t)+\beta_{3} O(t) \\
& \frac{d O(t)}{d t}=\alpha_{3} C_{3}(t)-\beta_{3} O(t)-\gamma O(t)+\delta I(t) \\
& \frac{d I(t)}{d t}=\gamma O(t)-\delta I(t)
\end{aligned}
$$

ecuacion 7

Lo que nos interesa es el estado $\mathrm{O}=\mathrm{O}(\mathrm{t})$ ya que este estado es el único que permite el paso de los iones generando corriente. 
Con lo anterior la ecuacion 4 queda como:

$C_{m} \frac{d(V(t))}{d t}=I-\left[G_{n a} O_{n a}\left(V(t)-V_{n a}\right)+G_{k} O_{K}\left(V(t)-V_{k}\right)+G_{l}\left(V(t)-V_{l}\right)\right]$ ecuación 8

Donde $O_{n a}$ y $O_{k}$ son las soluciones de $\mathrm{O}(\mathrm{t})$ para sodio y potasio de la ecuación 7. Con este sistema de ecuaciones se plantea que para que el canal se abra tiene que pasar por los estados cerrados y después al estado inactivado si es que este canal posee este estado.

\section{SIMULACIÓN}

Para resover la ecuación 8 tomamos los valores de $\alpha_{i}=\alpha_{i}(t, V), \beta_{i}=\beta_{i}(t, V), \gamma=\gamma(t, V), \delta=$ $\delta(t, V)$ reportadas por $\mathrm{H} \mathrm{y} \mathrm{H}$, usando estos valores se resuelve el sistema de ecuaciones 7 para determinar las corrientes de sodio y de potasio, una vez obteniendo las corrientes, se programó la resolución de la ecuación 8 y se determinó V=V(t). Todo esto se realizó con el software Wolfram Mathematica®.

\section{RESULTADOS DE LAS SIMULACIONES}

Al determinar la solución de la ecuación 8 , graficamos la función $\mathrm{V}=\mathrm{V}(\mathrm{t})$ y se pudo reproducir el PA reportado por $\mathrm{H}$ y $\mathrm{H}$ ver figura 2.

Figura 2: Simulación de Potencial de acción con el modelo. Se grafico la funcion $\mathrm{V}(\mathrm{t})$ de la solución de la ecuación 8 considerando los valores reportados experimentalmente de las corrientes de sodio y de potasio.

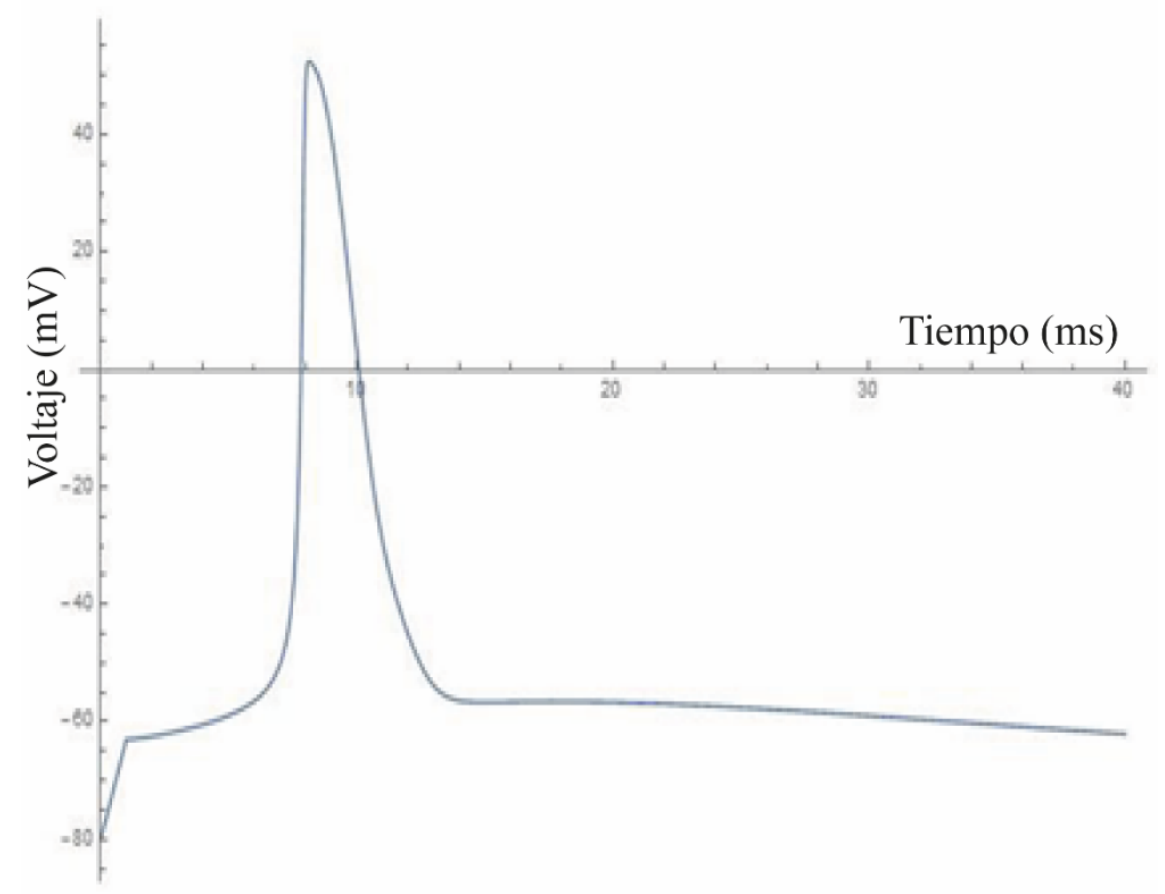




\subsection{PREDICCIONES}

Usando el modelo, propusimos aumento de la corriente de Potasio, los valores de esta corriente los aumentamos 1.771 y 1.772 veces, los resultados se muestran en la figura 3 a) y b) respectivamente. Al aumentar la corriente de potasio 0.001 veces ya no se produce el Potencial de Acción, ver figura 3 b.

Figura 3 Bloqueo de potencial de acción por aumento de corriente de potasio. El modelo presento alteraciones en la excitabilidad de la membrana por el aumento de la corriente de potasio en a) se aumento 1.771, y en b) el aumento fue de 1.772, nótese que en la condicion b) no se produce el potencial de acción.

a)

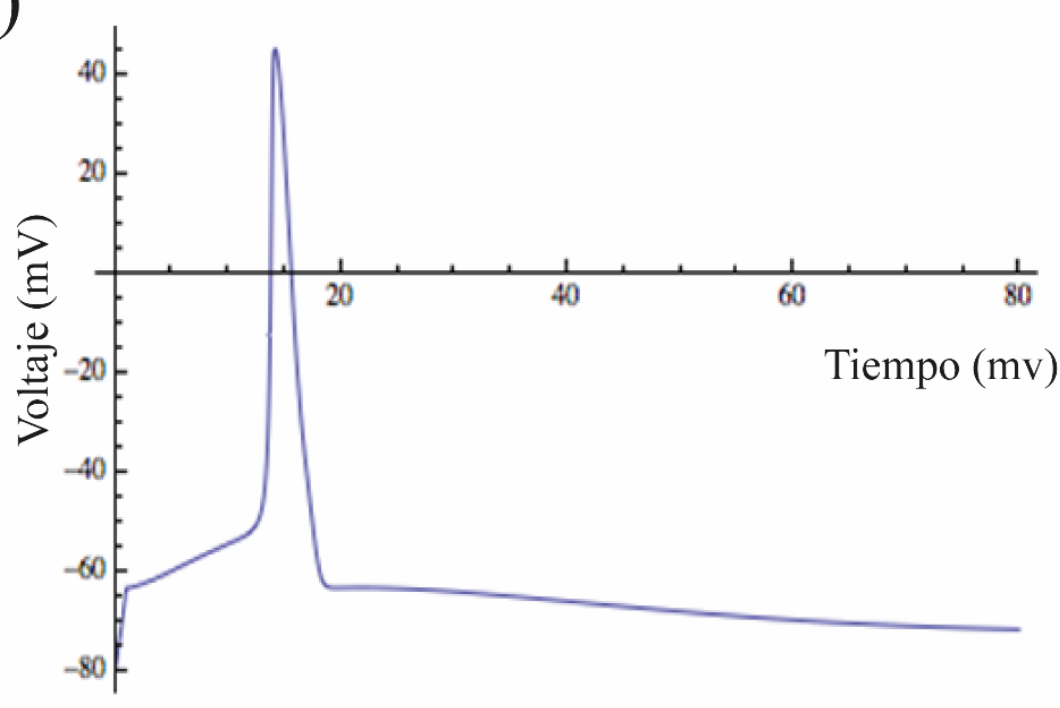

b)

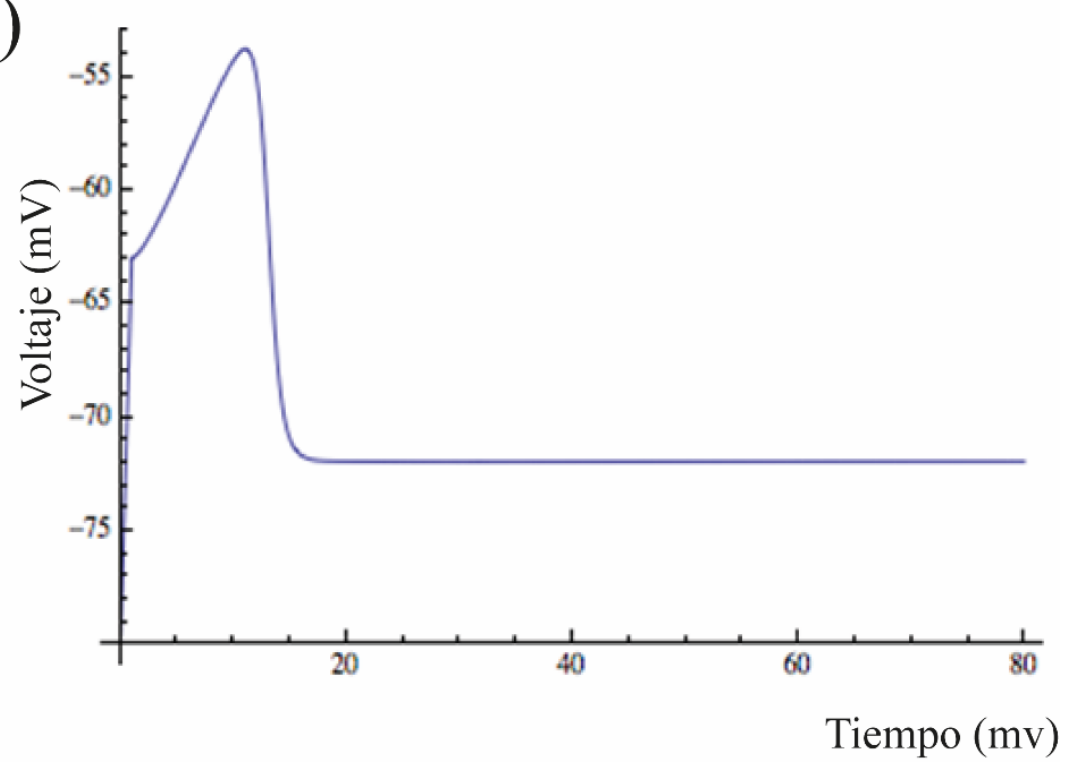

Sin embargo, al hacer cambios para las predicciones por un aumento en la corriente de sodio de 1.4, 1.5 y 1.58 veces (sin cambiar la de potasio), el aumento permitió observar alteraciones como pospotenciales tempranos ${ }^{7}$, estas simulaciones son difíciles de lograr, los resultados se muestran en la figura 4. 
Figura 4. El incremento de la corriente de sodio origina pospotenciales. En la gráfica se muestra los efectos del incremento de la corriente de sodio en a) el trazo del potencial de accion aumentado 1.4 en b) se aumenta 1.5 y en c) se aumentó 1.58 veces la corriente de sodio.

a)

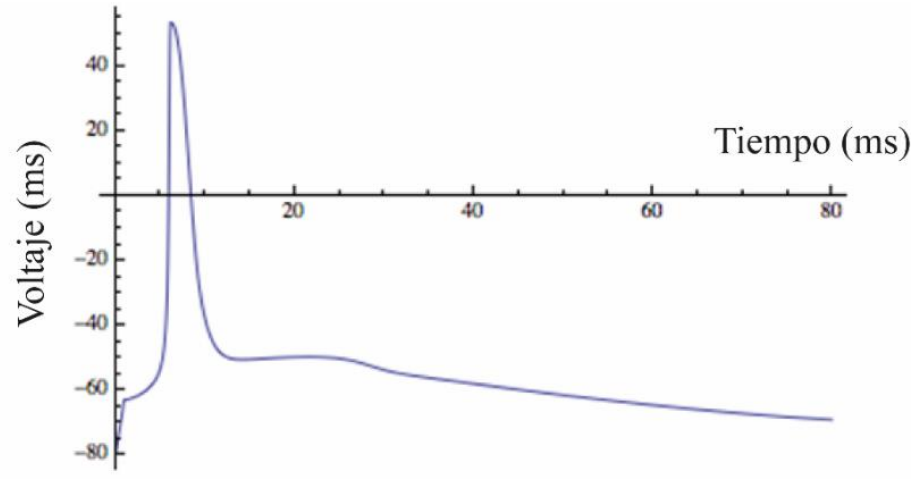

b)

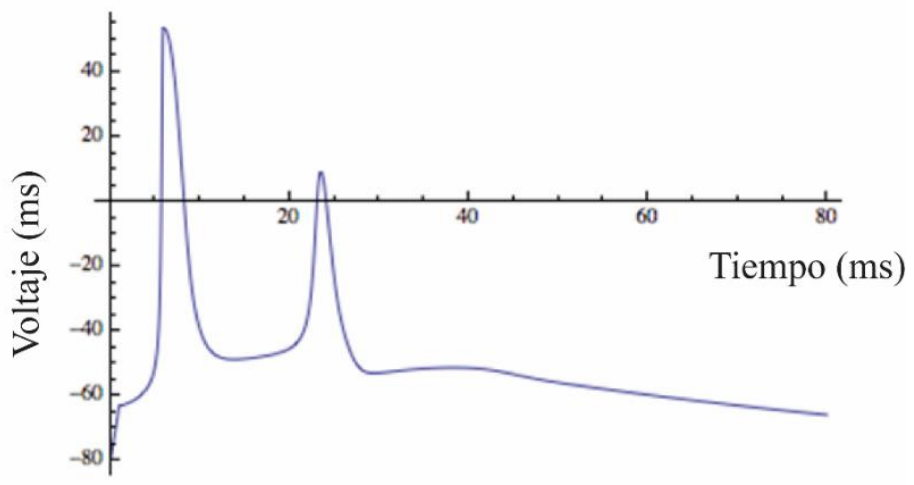

c)

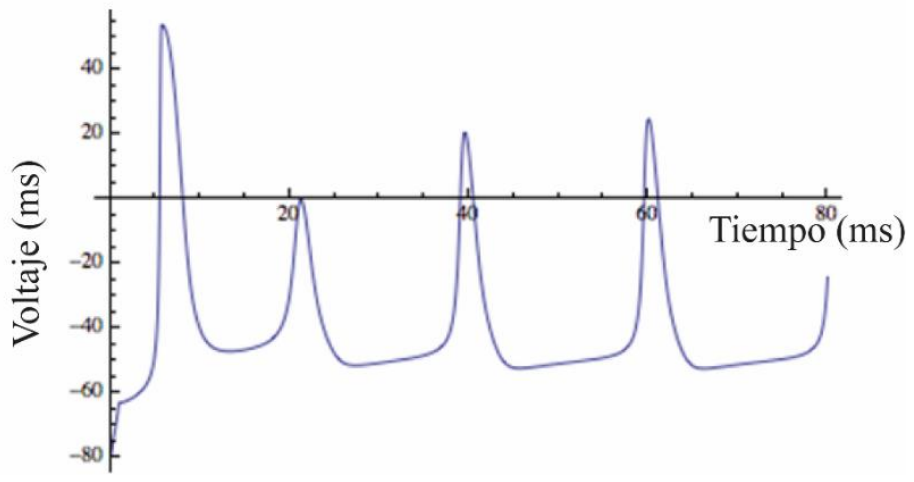

Nótese que cuando aumenta 1.4 veces la corriente de sodio se producen leves cambios al potencial de acción ya que solo entre los 20 y $22 \mathrm{~ms}$ se ve un aumento en el voltaje con respecto a la figura 2. Cuando la corriente de sodio aumenta 1.5 veces se nota la aparicion de un Potencial de Acción lento inciso b) figura 4, cuando aumenta 1.58 veces se nota la aparición de varios PA lentos inciso c) figura 4.

Una forma de aumentar la corriente de sodio es disminuir la velocidad de inactivación y esto lo simulamos al multiplicar este parámetro $\gamma$ por 0.1347 el resultado se presenta en la figura 5 . Notese que se presentan oxcilaciones en el potencial de acción en los intervalos de tiempo entre 22 y 60 ms. 
Figura 5. Alargamiento de la duración del potencial de acción. En la gráfica se observa un aumento en la duración por disminución en la velocidad de inactivación de la corriente de sodio. Nótese la aparición de oscilaciones en el potencial de acción.

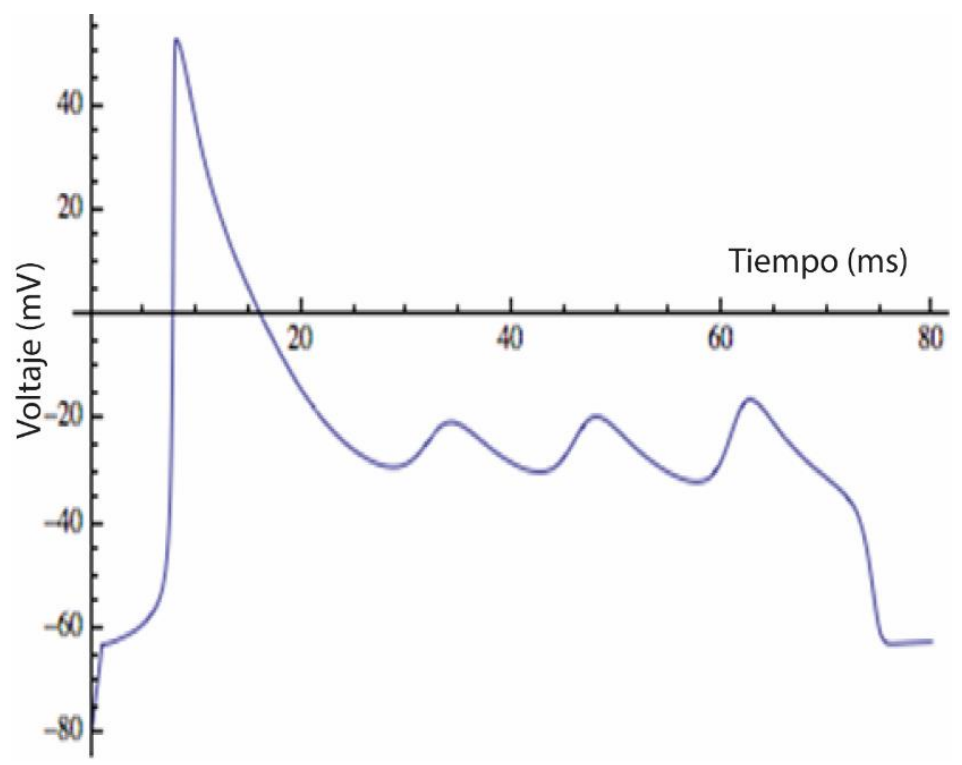

Otra forma de disminuir la corriente de sodio es aumentar la velocidad de recuperacion de la inactivacion $\delta$, esto se modelo al multiplicar este parametro por 1.8 el resultado se presenta en la figura 6.

Figura 6. Generación de los potenciales tempranos. En la gráfica se observa que al aumentar la velocidad de recuperación de la inactivación de la corriente de sodio se originan potenciales tempranos.

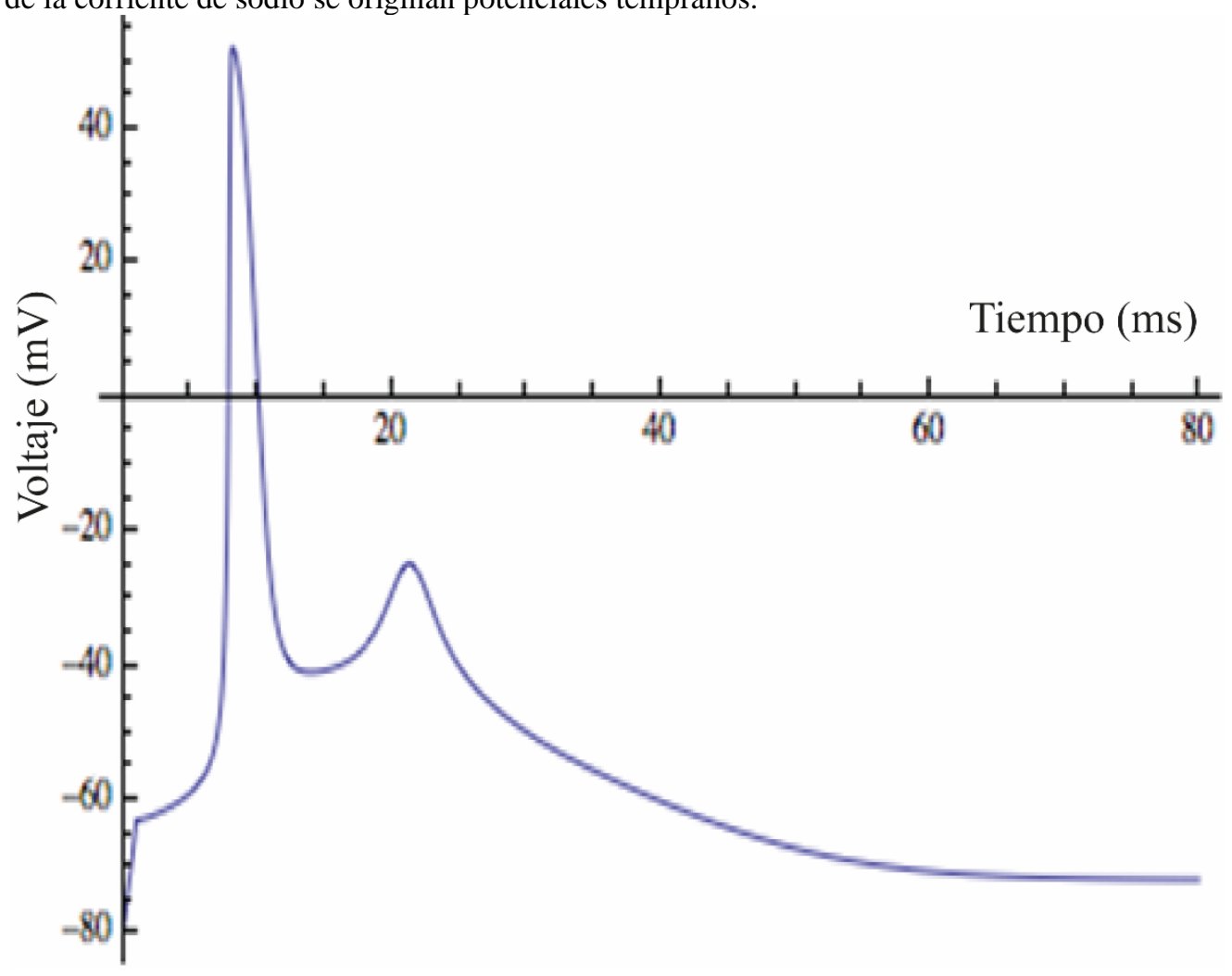


Se sabe que de la existencia de potenciales marcapasos y en estos participan las corrientes de sodio, potasio calcio tipo L, calcio tipo $\mathrm{T}$, Ikr, además de la corriente característica del marcapaso $\mathrm{I}_{\mathrm{f}}{ }^{8,9}$, decidimos resolver la ecuacion 8 sumando estas corrientes y el resultado se muestra en la figura 7, nótese que se obtuvo actividad eléctrica marcapaso.

Figura 7 Potencial de Acción tipo marcapaso. En esta grafica se observa la pendiente en la fase 4 del potencial de acción característica de la presencia de la corriente $\mathrm{I}_{\mathrm{f}}$.

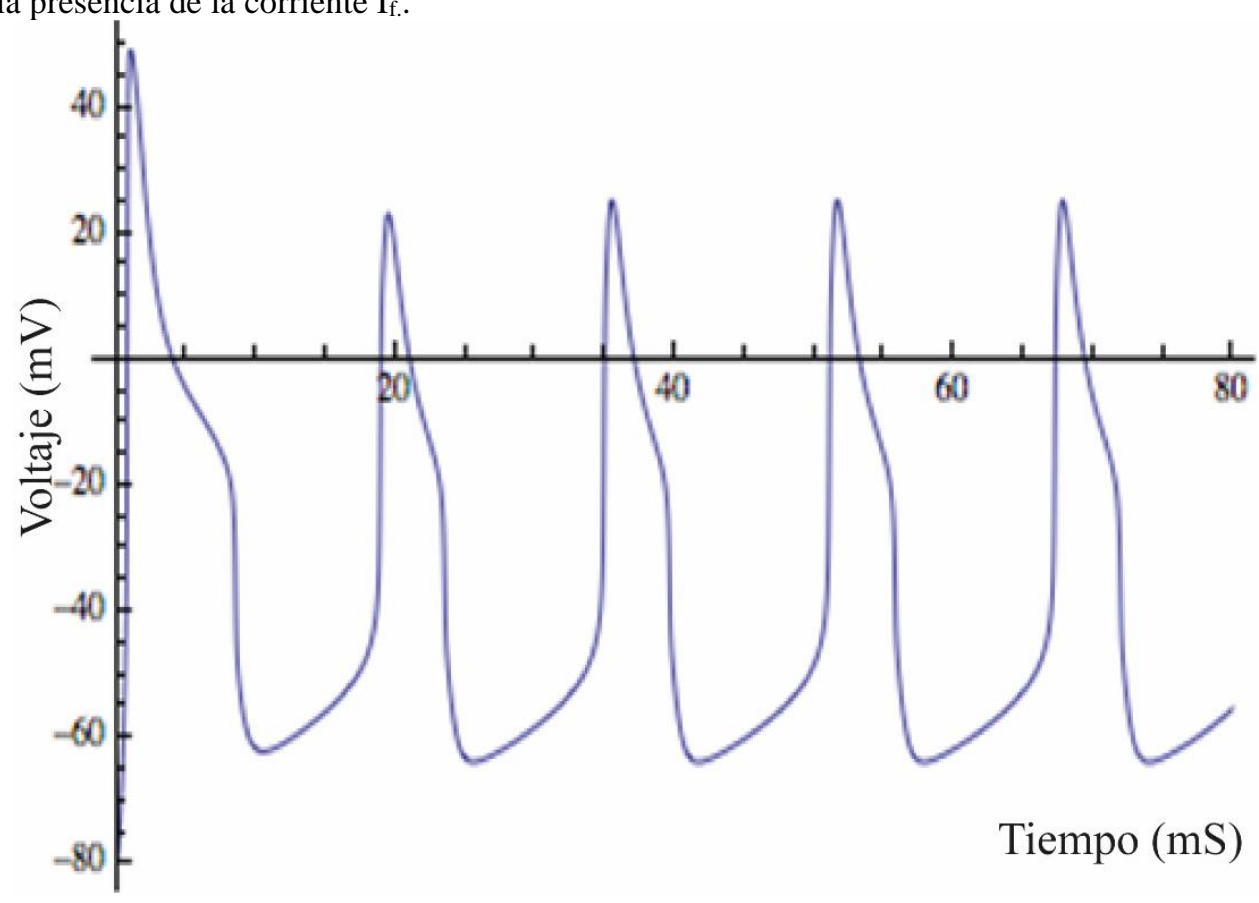

\section{DISCUSIÓN Y CONCLUSIONES}

En este trabajo se plantea que los canales ionicos se comportan como una reacción quimica (ecuación 6), por lo que se rigen por la ley de acción de masas con esta supocición se generan un sistema de ecuaciones diferenciales (ecuación 7) que se acoplan para resolver la ecuación 8, al determinar esta solución encontramos la función $\mathrm{V}=\mathrm{V}(\mathrm{t})$ que es el PA generado por las corrientes que se incluyen en la solucion.

Los PA generados son similares a los reportados por $\mathrm{H}$ y $\mathrm{H}$ en el caso de solo tomar en cuenta las corrientes de potasio y sodio. Por otro lado, usando este modelo analizamos la posibilidad de predecir alteraciones en el PA, usando alteraciones en la corriente de potasio y como resultado encontramos que cuando aumentamos 1.7 veces esta corriente, inciso a) figura 3, el potencial llega a un potencial de reposo cercano a los $-80 \mathrm{mV}$, es decir un voltaje menor al observado en la figura 2. Sin embargo, cuando la corriente de potasio se incrementa a 1.772 ya no se produce un PA solo se observa un aumento del voltaje de membrana, a valores que no permiten la apertura de los canales de sodio, por lo tanto, no aumenta la 
permeabilidad al ion sodio y no se produce el PA. Es decir, se describe una forma en cual la célula sea inexcitable, es decir, aumentando la corriente de potasio.

Por otro lado, si aumentamos la corriente de sodio a 1.4 veces su valor solo se observó un incremento del voltaje durante $22 \mathrm{~ms}$, por lo que el PA es similar al de la figura 2. Sin embargo, si el aumento de la corriente de sodio es de 1.5 veces aparece una despolarizacion que semeja un potencial lento, y cuando el valor es de 1.58 veces, el PA presenta potenciales lentos, semejantes a posdespolarizaciones tardías, que podrían propagarse generando lo que se conoce como un tren de disparo, esto garantizaría en el corazón arritmias de reentrada y en el sistema nervioso miotonías y parálisis periódicas ${ }^{10}$.

$\mathrm{Al}$ analizar los resultados de las alteraciones en las corrientes tanto de sodio como de potasio, propusimos aumentar las corrientes a través de la cinética de los canales, los resultados fueron similares a los del incremento de las corrientes. Lo primero que se hizo fue aumentar $\delta$, es decir alterar la velocidad de la recuperación de la inactivación del canal iónico. Ya que como se muestra en la ecuación 6, al aumentar esta velocidad el estado $\mathrm{O}(\mathrm{t})$ de inactivación aumenta la apertura del canal, al realizar esta operación encontramos que se generan unas pequeñas oscilaciones antes de terminar el potencial de acción. Este resultado permitió ver que al cambiar la cinética de los canales cambia la morfología del PA generando alteraciones patológicas.

Otra forma de aumentar la corriente de sodio es disminuir la velocidad de inactivación es decir la $\delta$, generando que el PA presente una despolarización al tiempo de los $21 \mathrm{~ms}$, generando también despolarizaciones lentas.

Las alteraciones en el PA fueron logradas con solo la multiplicación de un número a las velocidades de transición, lo cual facilita entender que los cambios de la morfología de los PA podrían ser por alteraciones en la cinética de los canales y que los problemas están también a nivel la cinética, lo anterior podría permitir un diagnóstico y pronóstico de las causas de las canalopatias.

Con los anteriores resultados, propusimos que las alteraciones que hicimos en la corriente de sodio pueden producir un potencial marcapaso, así que si las corrientes que se sabe que participan en los PA marcapasos generamos uno y al hacer esta suma nos encontramos que si es posible generar un potencial de acción marcapaso con nuestro modelo.

Otro aspecto que es importante remarcar es que en el modelo de $\mathrm{H}$ y $\mathrm{H}$ los parámetros n y $\mathrm{m}$ se tiene que elevar a la cuarta potencia y a la tercera potencia respectivamente, para que su modelo se ajuste al registrado experimentalmente, en este modelo al suponer que existen 3 estados cerrados como lo registrado experimentalmente, usando esta premisa no se tiene que multiplicar por ningún otro número, lo que representa una ventaja para entendimiento de la cinética y la caracterización de las corrientes de los canales y las canalopatías. 


\section{BIBLIOGRAFÍA}

1. Anumonwo JM, Tallini YN, Vetter FJ, Jalife J. Action potential characteristics and arrhythmogenic properties of the cardiac conduction system of the murine heart. Circ Res. 2001;89(4):329-335.

2. Levin KH, Chauvel P. Preface. Handb Clin Neurol. 2019;160:ix.

3. Hodgkin AL, Huxley AF. Propagation of electrical signals along giant nerve fibers. Proc $R$ Soc Lond B Biol Sci. 1952;140(899):177-183.

4. Barry DM, Nerbonne JM. Myocardial potassium channels: electrophysiological and molecular diversity. Anпu Rev Physiol. 1996;58:363-394.

5. Cole KS, Curtis HJ. Electric Impedance of the Squid Giant Axon during Activity. J Gen Physiol. 1939;22(5):649-670.

6. Julian Torres-Jacome JMM-D, Areli Montes-Perez, Guadalupe Montiel-Jaen, Otto Garcia Garibay, Moran- Raya Carolina, Albarado-Ibañez Alondra. Macroscopic currents of ionic channels using Mass Action Law: A mathematical model: . South Florida Journal of Development, Miami, . 2021 2(5):7493.

7. Ghosh S, Cooper DH, Vijayakumar R, Zhang J, Pollak S, Haissaguerre M, Rudy Y. Early repolarization associated with sudden death: insights from noninvasive electrocardiographic imaging. Heart Rhythm. 2010;7(4):534-537.

8. Albarado-Ibanez A, Avelino-Cruz JE, Velasco M, Torres-Jacome J, Hiriart M. Metabolic Syndrome Remodels Electrical Activity of the Sinoatrial Node and Produces Arrhythmias in Rats. Plos One. 2013;8(11).

9. Kurata Y, Matsuda H, Hisatome I, Shibamoto T. Effects of pacemaker currents on creation and modulation of human ventricular pacemaker: theoretical study with application to biological pacemaker engineering. American Journal of Physiology-Heart and Circulatory Physiology. 2007;292(1):H701H718.

10. Catterall WA. Dravet Syndrome: A Sodium Channel Interneuronopathy. Curr Opin Physiol. 2018;2:42-50. 